\title{
Psychophysiological effects of practicing static qigong meditation in breast cancer survivors
}

\author{
Georgel Arhip, Ion Dafinoiu, Gabriela M. Assante, \\ Dan V. Filimon, Dănuţ C. Irimia
}

Arhip Georgel - PhD Researcher, Alexandru Ioan Cuza University, Faculty of Psychology and Educational Sciences, Toma Cozma Street 3, Iași, 700554

Ion Dafinoiu - Professor, PhD, Alexandru Ioan Cuza University, Faculty of Psychology and Educational Sciences, Toma Cozma Street 3, Iași, 700554

Gabriela Monica Assante- PhD, Alexandru Ioan Cuza University, Faculty of Psychology and Educational Sciences, Toma Cozma Street 3, Iași, 700554

Dan Vlad Filimon - MD PhD, Specialist in Traditional Chinese Medicine, Acupuncture Practitioner, Orhei Street 8, Piatra Neamţ, 610257

Dănuţ Constantin Irimia - PhD, Gheorghe Asachi Technical University of Iaşi, Faculty of Electrical Engineering, Energetics and Applied Informatics, Dimitrie Mangeron Street 67, Iaşi, 700050

\section{ABSTRACT}

Background: Cancer treatment can result in severe impairments regarding patients quality of life, such as persistent fatigue, sleep disturbance, and a series of psychological conditions ranging from fear or sadness to anxiety or even depression. Various forms of meditation have been studied in association with the reduction of symptoms related to cancer and cancer therapy. The growing number of breast cancer survivors triggers the need of a better attention in clinical practice regarding their quality of life.

Objectives: The aim of the present research consists in investigating the extent to which meditation, specifically, static qigong as a psychotherapeutic method, is effective in ameliorating impairments in breast cancer survivors.

Method: Thirty-one breast cancer survivors participated in the research. The subjects that never practiced meditation were invited to join a research during which they had to practice static qigong meditation, once a day, for five weeks. Several psychophysiological indicators such as, plasma cortisol level, electrodermal reaction, palms temperature, oxygen saturation in the blood, anxiety levels, illness perception were measured before and after the intervention, and social support and personality features before intervention.

Results: The results of the paired samples $t$-tests suggest that after the five-week intervention lower cortisol levels were obtained, as well as a better electrodermal reaction, increased palms 


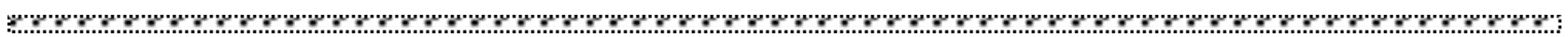
temperature and better oxygen saturation in the blood. The anxiety levels decreased and a more positive illness perception resulted after the five-weeks study.

Conclusion: The outcomes of the current research indicate that breast cancer survivors can benefit from an improved health as a result of practising static qigong meditation. .

\section{KEYWORDS:}

\section{Breast cancer survivors, static qigong, psychophysiological effects}

\section{INTRODUCTION}

Cancer with its debilitating symptoms as well as cancer treatment contribute to significant decline in subjective and objective wellbeing. Breast cancer survivors can have persistent fatigue, sleep disturbance, and a series of psychological conditions ranging from fear or sadness to anxiety or even depression. However, there is insufficient evidence to recommend certain interventions as standard care. Interventional studies are needed to provide the necessary evidence to inform health care professionals' practice for meeting the needs of breast cancer survivors. Various forms of physical activity and meditation have been studied in association with the reduction of symptoms related to cancer and cancer therapy $(1,2)$. The growing number of breast cancer survivors triggers the need of a better attention in clinical practice regarding their quality of life. The evidence suggest that the main cause of these symptoms is the complex of stress responses post cancer diagnosis and treatment including sleep disorders, anaemia, and inflammation (2). It is possible for some of these symptoms to co-occur because of the underlying inflammatory biomarker changes which are generally associated with fatigue, depression or sleep disorders (3).

In general, meditation and consequently qigong practice, are regarded as complementary or alternative therapies to various health related conditions. As a mindbody intervention based on energy therapy, it is founded on the assumption that there are various energy fields around the human body.
It is considered that by guiding the claimed energy field by visualization and meditation can result in an improved health status (4). Energy (qi) can be directly guided by consciousness and breathing. Making use of guiding skills, qigong can integrate one's body, mind, and breath adjusting the body's energy system. From the qigong practice perspective, various medical conditions are triggered from a misaligned and imbalanced energy. This energy can be adjusted to a normal state through unifying the body, mind and breath, hence, by practicing qigong (5). For example, Barabasz (6) have obtained spectacular results in a patient with cancer by applying a static qigong visualization protocol. The energy system cannot be directly observed because it has no clear boundaries, but it can be induced by consciousness, specifically through the Microcosmic Orbit of qigong practice. The method entails two paths through which energy (qi) mouves through body. For this reasons, it is extremely difficult to apply the assumptions that underlie the experimental methods which are generally used in empirical research. Qigong consists of two practice styles: static and dynamic (7). The static form is purely meditative without the exercise component, including visualization exercises of the moving energy (Microcosmic Orbit). Consequently, the dynamic includes the exercise component (8).

As mentioned before, meditation appears to be a valuable complementary measure for overcoming the long-lasting impairments in the case of breast cancer survivors. Several 
(n)

studies have proven that qigong has beneficial effects in promoting a good quality of life, reduced fatigue, improved sleep as well as better cognitive function. Regarding the quality of life it must be stressed that a twomonth protocol could improve the quality of life and decrease distress. Moreover, if it takes place in a group format, the cancer survivors can support each other (9). The same benefits related to their significantly improved mental health and quality of life status after a short period of practice was also observed by a group of Brazilian researchers (10). In addition they stress that a prolonged period of practice did not increased the initial results. Furthermore, an extensive trial concluded by saying that practicing qigong leads to a better quality of life in the interventional group when compared to placebo or control group (11). Therefore, meditative static qigong may be used to promote health and to reduce all-cause mortality. In the survivorship phase contributes to a better self-management of health and wellbeing. The practice of qigong also resulted in decreased fatigue even three months after the intervention in breast cancer survivors (12). Better quality of sleep was observed in the same group after a six-week intervention (13). These benefits are supported by various other studies which suggest that static qigong as a mental practice includes a significant degree of internalized attention that correlates with theta activity. In qigong practitioners was observed an increase in alpha activity which is related to increased relaxation and well-being. As well as an increase of EEG frontal theta activity, associated to an attentive state which is one of the reaching objectives in meditation. Meditation-based interventions demonstrate efficacy in reducing stress, behavioral symptoms, and proinflammatory signaling in breast cancer survivors $(14,15)$. Although the functioning mechanisms of qigong practice have not been yet elucidated, some findings suggests two possible explanations: qigong practice focusing on meditation and deep breathing may increase melatonin $(16,17)$ and may also affect stress response pathway $(18,19)$.

The aim of the present research was to examine the outcomes of static qigong meditation in breast cancer survivors. The main interest lies in changes in plasma cortisol levels, electrodermal reaction, palms temperature and oxygen saturation in the blood following, five weeks after the start of qigong meditation. In addition, outcome measures included various psychological variables such as anxiety levels, illness perception social support and certain personality features. Participants' trust in therapist and in intervention was also included. We expect to find improvements with respect to the variables related to quality of life.

\section{METHOD}

The central objective of the present research is to investigate the extent to which meditation, specifically, static qigong as a psychotherapeutic method, is effective in ameliorating impairments in breast cancer survivors. Some protocols of qigong practice (e.g. Microcosmic Orbit) are easy to learn and require little physical capacity to practice, thus making it more desirable to patients who are less physically capable. The physiological indicators central to this study are: plasma cortisol level (nmol/L), indicating the level of stress that affects the functioning of the immune system; electrodermal reaction (skin resistence, $\mathrm{M} \Omega$ ), measured by using a digital ohmmeter, indicates muscle and mental relaxation; palms temperature (Celsius degrees), evaluated with a thermovision camera, it shows the peripheral blood circulation; oxygen saturation in the blood $(\%)$, measured with a pulseoximetry device 


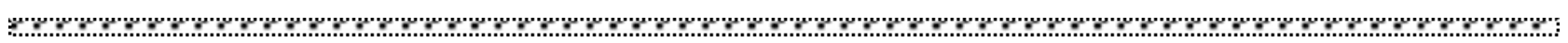
indicates the lungs capacity to provide oxygen for normal body function.

The main psychological measures used evaluated the anxiety levels, illness perception, social support and personality features. The anxiety was measured using State-Trait Anxiety Inventory (STAI-X1 form), which is considered one of the most widely used anxiety measures (20). A satisfying Cronbach's $\alpha$ value of .77 was obtained. The form used in the present study underlines the changeable state components of anxiety.

Illness perception was evaluated through the means of Brief Illness Perception Questionnaire (Brief IPQ) which combines eight items for measuring cognitive illness representations (consequences, timeline, personal control, treatment control, and identity), emotional representations (concern and emotions related to the illness) and illness comprehensibility (21). Alpha Cronbach in this case was .79.

The used measure for social support was The Berlin Social Support Scales (BSSS). This particular instrument was developed based on theoretical considerations and reviews of other measures for social support. It consists of 32 items organized on four subscales where a sum score can be calculated (22). In the present study alpha was .69.

The Five-Factor Model Rating Form (23) was used for measuring several personality features. It is a rating form consisting of 30 facets of the five-factor model. The 30 items are organized with respect to five domains: openness to experience, conscientiousness, extraversion, agreeableness and neuroticism. Cronbach's $\alpha$ values for the domains within the sample ranged from .65 (openness to experience) to .78 (conscientiousness), with a median of .71 .

The described indicators were measured before the start of the intervention and five weeks after the participants started practicing meditation. Therefore, after obtaining the Ethical Committee Approval and the signed consent form from the participants the aim of the research was further explained, as well as the underlying assumptions of static qigong meditation Microcosmic Orbit. The participants filled out the questionnaires. Subsequently the measuring devices were installed and the participants started listening to an audiotaped qigong meditation exercise (Microcosmic Orbit). They received the recording at home together with the recommendation to practice this form of meditation daily at home, until the end of the study.

\section{RESULTS}

\section{PARTICIPANTS}

A total of 31 breast cancer survivors, with 2 to 20 years after the mastectomy surgery, participated in the research. All participants have partial or total mastectomy. The participants age range between 40 and 72 years old, with an average age of 61 years old. The subjects have never practiced meditation. The decision to include in the research only novice is based on the intention to provide a therapeutic method that may contribute to an improved health status, which is easy to practice and available to everyone. Thirty-six participants were initially recruited, but five were excluded for various reasons: two of them did not perform the cortisol test at the posttest stage, other two did not practice meditation for the indicated period, and one participant mentioned in the post-testing phase that was practicing Zen meditation for at least 10 years. None of the participants had chemotherapy or radiotherapy treatment during the research. No participant reported any adverse effect during the research. 


\section{EFFECTIVENESS OF THE} INTERVENTION

Paired-samples t-tests were performed in order to investigate the effectiveness of the intervention. Moreover, independent samples t-tests were used in order to see if the observed effect can be attributed to the intervention or to other variables. A pairedsamples $t$-test was conducted to compare the plasma cortisol levels before the intervention and five weeks after starting static qigong practice. There was a significant difference in the pre-test $(\mathrm{M}=525.18, \mathrm{SD}=91.31)$ and posttest $\quad(\mathrm{M}=440.09, \quad \mathrm{SD}=80.21) \quad$ conditions; $t(30)=-10.56, p=0.000$. These results suggest that cortisol levels decreased postintervention. The same significance was obtained in the case of electrodermal reaction. There is a difference regarding the electrodermal reaction before the intervention $(\mathrm{M}=0.44, \quad \mathrm{SD}=0.27)$ and post-intervention $(\mathrm{M}=0.61, \mathrm{SD}=0.41)$ conditions; $\mathrm{t}(30)=-2.43$, $\mathrm{p}=0.021$, suggesting that post-intervention a higher electrodermal resistance was obtained. Regarding the palms temperature the results show an significant increase when the pre-test $(\mathrm{M}=34.33, \mathrm{SD}=1.00)$ and post-test $(\mathrm{M}=35.15$, $\mathrm{SD}=0.64)$ conditions are compared; $\mathrm{t}(30)=-$ 4.26, $\mathrm{p}=0.000$. The evidence shows an increase of the average palms temperature after the five-week intervention. The oxygen saturation in the blood before intervention $(\mathrm{M}=91.06, \mathrm{SD}=2.42)$ is significantly lower than post-intervention $(\mathrm{M}=94,77, \mathrm{SD}=2.02)$ condition; $\mathrm{t}(30)=-10.56, \mathrm{p}=0.000$, suggesting a better oxygenation after the intervention.

The participants showed an improvement in their illness perception. The first measurement $(\mathrm{M}=32.09, \mathrm{SD}=2.13)$ resulted in higher scores when compared with the second measurement $\quad(\mathrm{M}=29.19, \quad \mathrm{SD}=1.80)$, suggesting a significant progress $(\mathrm{t}(30)=$ $3.78, \mathrm{p}=0.001$ ) towards a more positive illness perception. The anxiety levels during pre-test
$(\mathrm{M}=51.23, \mathrm{SD}=11.31)$ when compared to the post-test $\quad(\mathrm{M}=44.44, \quad \mathrm{SD}=9.40) \quad$ where significantly higher $(\mathrm{t}(30)=6.83, \mathrm{p}=0.000)$ indicating that the participants progressed towards a less anxious state (Table I).

Table 1. Comparisons of the changes in the outcome measures before and after the intervention

\begin{tabular}{|c|c|c|c|c|c|c|}
\hline \multirow{3}{*}{$\begin{array}{l}\text { Outcome } \\
\text { variables }\end{array}$} & Time & & & & t-test & p \\
\hline & $\begin{array}{l}\text { Pre- } \\
\text { test }\end{array}$ & & \multicolumn{2}{|c|}{ Post-test } & & \\
\hline & Mean & SD & Mean & SD & & \\
\hline $\begin{array}{l}\text { Cortisol } \\
(\mathrm{nmol} / \mathrm{L})\end{array}$ & 525.18 & 91.31 & 440.09 & 80.21 & $\begin{array}{l}- \\
10.561\end{array}$ & $.000 *$ \\
\hline $\begin{array}{l}\text { Electrodermal } \\
\text { reaction }(\mathrm{M} \Omega)\end{array}$ & .44 & .27 & .61 & .41 & -2.43 & $.021^{*}$ \\
\hline $\begin{array}{l}\text { Palms } \\
\text { temperature } \\
\left({ }^{\circ} \mathrm{C}\right)\end{array}$ & 34.33 & 1 & 35.15 & .64 & -4.26 & $.000 *$ \\
\hline $\begin{array}{l}\text { Oxygen } \\
\text { saturation in } \\
\text { the blood }(\%)\end{array}$ & 91.06 & 2.42 & 94.77 & 2.42 & -10.56 & $.000 *$ \\
\hline $\begin{array}{l}\text { Illness } \\
\text { perception }\end{array}$ & 32.09 & 2.13 & 29.19 & 1.80 & 3.78 & $.000^{*}$ \\
\hline Anxiety & 51.23 & 11.31 & 44.44 & 9.40 & 6.83 & $.000 *$ \\
\hline
\end{tabular}

* Significant differences are reported at the $\mathrm{p}$ $<.05$ level

We wanted to establish whether or not the obtained evidence are a result of the intervention. Therefore, independent samples $t$-tests were performed to verify if the cortisol levels are influenced by some personality features, social support and patients believes in the therapist or static qigong. The results showed that in the post-hoc phase the group which confide the most in the efficiency of static qigong benefited from lower cortisol levels $(\mathrm{t}(29)=2.33, \mathrm{p}=0.027)$. Also, the group with high confidence in the therapist positively influenced the post-test cortisol levels $(\mathrm{t}(29)=2.24, \mathrm{p}=0.035)$. The personality traits and social support showed no effect on the post-test measurements.

\section{DISCUSSION}

The imaginary route of static qigong meditation, specifically the microcosmic orbit technique, overlaps the acupuncture meridian 
(1)

circuit concept vessel and governor vas, which are morpho-functional structures made up of connective tissues with pronounced bioelectric properties such as piezo and pyroelectricity, aspects that explain the phenomena of acupuncture $(24,25)$. The electrical activity of these morpho-functional structures is influenced by brain activity during a "mind-body dialogue" (26). Its fundamental role lies in maintaining the biosystem homeostasis called the human body. Arnold van Loof (26) shows that the difficulty of explaining the mind-body relationship, in which the body is associated with the "material aspect" of living beings, and the mind with the "immaterial" (consciousness) can be overcome because the aforementioned relationship is essential to physiological mechanisms. The argument is of an electrical nature in the sense that in any living being, the cellular electrical component is vital, the death of any cell occurring when it irrevocably loses its ability to provide its own electrical energy. Electricity is basically exchange of immaterial information (e.g. zero mass). As is well known, the brain operates with variations in electrical values. It is also important to note that the brain does not distinguish between electrical signals from the real world and those produced by ones' own imagination. Both types of signals are of an electrical nature, they are decoded as information, zero mass entities that provide communication between physical and nonphysical. The fact that bioelectric activities are amended through meditation, as a mental exercise, produces electrical signals that the brain processes to restore the electrophysiological activities of the cell, which in turn potentiates the electrical homeostasis that ensures the maintenance of the entire biosystem.

In our research, this mind-body dialogue influences the physiological parameters proposed for measurement. Thus, serum cortisol levels have decreased significantly, indicating a tendency for normal immune system functioning by reducing the intensity of chronic inflammatory processes (27). This effect results from the rebalancing of the vegetative balance in favor of the parasympathetic component, known as the fact that in the meditative process there is a significant decrease in sympathetic nervous system induced catabolic activity $(28,29,30$, $31,32)$. Oxygen concentration in peripheral blood has increased, indicating a predominance of parasympathetic activity. The state of parasympathicotonia is induced by the activation of the vagus nerve whose visceroperipheral distribution ensures the regeneration and functioning of extremely important tissues in the concept of homeostasis (33). Electrodermal reaction (RED), highlighted in our research as the electrical resistance of the skin, is a psychophysiological index that reflects changes in sympathetic autonomic nervous system activities that are assimilated to emotional and cognitive states (34). RED is probably the most useful index of changes induced by sympathetic excitation because it is the only autonomic psychophysiological variable not contaminated by parasympathetic activity (35). This is the reason for this parameter is used in polygraph technique. Determining this parameter in our research has undoubtedly highlighted the significant decrease in the activity of the sympathetic component of the autonomic nervous system as a result of practicing meditation. The thermal parameter (palm temperature) supplements the information on neurovegetative recalibration by the predominance of parasympathetic activity, as we have previously shown in the oxygen concentration parameter in peripheral blood (33).

In terms of reducing the state of anxiety, research shows that meditation can regulate 
(n)

emotions by strengthening the top-down cognitive control mechanisms of cerebral tonsil (36). At experienced practitioners, diminished activity in the amygdala was observed in response to emotional stimuli (37). Another study, which examines the tonsil response to emotional images in meditation, found that the tonsil reactivity was reduced and that top-down control between the pre-frontal and tonsillar cortex was strengthened (38). However, a lower reactivity of the tonsil determines a lower hormonal response to the hypothalamuspituitary gland-adrenal glands (HPA) axis. Therefore, it results in a lower level of cortisol and norepinephrine, which reduces anxiety and improves the immune system response $(39,40)$.

One of the limitations that might be imputed to this research consists in failing to determine the mechanisms through which static qigong practice produces this type of changes. The object of study is the energy which is difficult to study in traditional experimental settings due to unclear boundaries (5). The results suggest that the participants with high beliefs in the efficiency of static qigong and in the therapist had lower cortisol levels. This strongly suggests that the importance of the therapeutic relationship must not be ignored. It is very well known that the placebo effect should not be limited only to medicinal treatments; it certainly can be extended to the therapeutic relationship itself (41). For example several authors have argued that placebo effects could probably derive more from the individual belief in the qualities of the therapist than from the treatment itself $(41,42,43)$. There is a clear support of this assumption, ones of the lead authors in the field stating that the strong belief in the positive outcome of the therapy associated with the social influence brought by the therapist, represent fundamental placebo factors in any successful therapy (43, 44). The control group was not included in the research because the main scope was for everyone to benefit from the intervention.

Is it true that the evidence regarding the potential placebo effect of the therapist is scarce, when compared to the placebo effect as part of efficacy trials with certain treatments. Also, is just as true that there are no evidence which state that the benefits of the therapies should be attributed completely to the placebo effect (44). In conclusion, it must be stressed that when practicing any type of therapeutic protocol one must consider the importance of all related factors. By doing so, it might increase the expected outcomes.

\section{ACKNOWLEDGEMENTS AND DISCLOSURES}

The authors declare that they have no potential conflicts of interest to disclose

\section{REFERENCES}

1. Asher A, Myers JS. The effect of cancer treatment on cognitive function. Clinical advances in hematology \& oncology: H\&O. 2015; 13(7):441-50.

2. Myers JS. Review complementary and integrative interventions for cancer-related cognitive changes. Asia-Pacific journal of oncology nursing. 2015; 2(4):215.

3. Knobf MT, Thompson AS, Fennie K, Erdos D. The effect of a community-based exercise intervention on symptoms and quality of life. Cancer nursing. 2014; 37(2):43.

4. Cassileth BR, Deng G. Complementary and alternative therapies for cancer. The oncologist. 2004; 9(1):80-9.

5. Liu T. The scientific hypothesis of an "energy system" in the human body. Journal of Traditional Chinese Medical Sciences. 2018;5(1):29-34. 
r.p.

6. Barabasz AF, Barabasz M, Watkins JG. Single-session manualized ego state therapy (EST) for combat stress injury, PTSD, and ASD, Part 1: The theory. International Journal of Clinical and Experimental Hypnosis. 2011 Oct 1;59(4):379-91.

7. Tsang HW, Cheung L, Lak DC. Qigong as a psychosocial intervention for depressed elderly with chronic physical illnesses. International journal of geriatric psychiatry. 2002;17(12):1146-54.

8. $\mathrm{Ng} \mathrm{BH}$, Tsang HW. Psychophysiological outcomes of health qigong for chronic conditions: a systematic review. Psychophysiology. 2009;46(2):257-69.

9. Loh SY, Lee SY, Murray L. Study protocol to investigate the efficacy of participation in Qi-Gong by breast cancer survivors. Journal of Cancer Therapy. 2011:2.

10. Castellar, J. I., Fernandes, C. A., \& Tosta, C. E. (2014). Beneficial effects of pranic meditation on the mental health and quality of life of breast cancer survivors. Integrative cancer therapies, 13(4), 341-350.

11. Loh SY, Lee SY, Murray L. The Kuala Lumpur Qigong trial for women in the Cancer Survivorship phase-efficacy of a three-arm RCT to improve QOL. Asian Pacific Journal of Cancer Prevention. 2014;15(19):8127-34.

12. Larkey LK, Roe DJ, Weihs KL, Jahnke R, Lopez AM, Rogers CE, Oh B, Guillen-Rodriguez J. Randomized controlled trial of Qigong/Tai Chi Easy on cancer-related fatigue in breast cancer survivors. Annals of Behavioral Medicine. 2014; 49(2):165-76.

13. Liu W, Schaffer L, Herrs N, Chollet C, Taylor S. Improved sleep after Qigong exercise in breast cancer survivors: A pilot study. Asia-Pacific journal of oncology nursing. 2015; 2(4):232.

14. Bower JE, Crosswell AD, Stanton AL, Crespi CM, Winston D, Arevalo J, Ma J, Cole SW, Ganz PA. Mindfulness meditation for younger breast cancer survivors: a randomized controlled trial. Cancer. 2015; 121(8):1231-40.

15. Matchim Y, Armer JM, Stewart BR. Mindfulness-based stress reduction among breast cancer survivors: a literature review and discussion. Oncology nursing forum $2011 ; 38(2)$.

16. Martarelli D, Cocchioni M, Scuri S, Pompei P. Diaphragmatic breathing reduces exercise-induced oxidative stress. Evid Based Complement Alternat Med 2011; 932430.

17. Kim DH, Moon YS, Kim HS, Jung JS, Park HM, Suh HW, et al. Effect of Zen meditation on serum nitric oxide activity and lipid peroxidation. Prog Neuropsychopharmacol Biol Psychiatry 2005;29:327-31.

18. Lee MS, Lee MS, Kim HJ, Moon SR. Qigong reduced blood pressure and catecholamine levels of patients with essential hypertension. Int J Neurosci 2003;113:1691-701.

19. Jones BM. Changes in cytokine production in healthy subjects practicing Guolin Qigong: A pilot study. BMC Complement Altern Med 2001;1:8.

20. Spielberger CD, Gorsuch RL, Lushene RE. State-trait anxiety inventory manual. Mind Garden, Inc. 1970.

21. Broadbent E, Petrie KJ, Main J, Weinman J. The brief illness perception questionnaire. Journal of psychosomatic research. 2006; 60(6):631-7.

22. Schwarzer R, Schulz U. Berlin social support scales (BSSS). 2000; 6.

23. McCrae RR, Costa PT. Validation of the five-factor model of personality across instruments and observers. Journal of personality and social psychology. 1987; 52(1):81.

24. Filimon, DV, Contribuții la explorarea electrofiziologică, termografică și histochimică a zonelor de acupunctură, lucrare în curs de publicare.

25. Langevin HM, Nedergaard M, Howe AK. Cellular control of connective tissue matrix tension. Journal of cellular biochemistry. 2013; 114(8):1714-9.

26. De Loof A. The cell's self-generated "electrome": The biophysical essence of the immaterial dimension of Life?. Communicative \& integrative biology. 2016; 9(5):e1197446.

27. Rupprecht R, Wodarz N, Kornhuber J, Schmitz B, Wild K, Braner HU, Müller OA, Riederer P. In vivo and in vitro effects of glucocorticoids on lymphocyte proliferation in man: relationship to glucocorticoid receptors. Neuropsychobiology. 1990; 24(2):61-6.

28. Lehrer PM. Psychophysiological effects of progressive relaxation in anxiety neurotic patients and of progressive relaxation and alpha feedback in nonpatients. Journal of Consulting and Clinical Psychology. 1978; 46(3):389.

29. Benson H., Klipper MZ. The Relaxation Response. New York: William Morrow and Co.

30. Sargent JD, Green EE, Walters ED. Preliminary report on the use of autogenic feedback training in the treatment of migraine and tension headaches. Psychosomatic Medicine. 1973.

31. Schultz JH: Das Autogene Training. Leipzig: G. Thieme. 1932.

32. Schultz JH, Luthe W. Autogenic Training: A Psychophysiological Approach in Psychotherapy, New York: Grune \& Stratton. 1959.

33. Kasper DL, Braunwald E, Hauser S, Longo D, Jameson JL. Harrison's Principles of Internal Medicine 16th Edition. McGraw-Hill, 2004

34. Nagai Y, Critchley HD, Featherstone E, Trimble MR, Dolan RJ. Activity in ventromedial prefrontal cortex covaries with sympathetic skin conductance level: a physiological account of a "default mode" of brain function. Neuroimage. 2004; 22(1):243-51.

35. Dawson ME, Schell AM, Filion DL. The electrodermal system. Handbook of psychophysiology. 2007; 2:200-23.

36. Brandmeyer T, Delorme A, Wahbeh H. The neuroscience of meditation: classification, phenomenology, correlates, and mechanisms. Progress in brain research. 2019; 244:1-29. 
37. Tang YY, Hölzel BK, Posner MI. The neuroscience of mindfulness meditation. Nature Reviews Neuroscience. $2015 ; 16(4): 213$.

38. Kral, T. R., Schuyler, B. S., Mumford, J. A., Rosenkranz, M. A., Lutz, A., \& Davidson, R. J. (2018). Impact of short-and long-term mindfulness meditation training on amygdala reactivity to emotional stimuli. Neuroimage, 181, 301-313.

39. Esterling BA, Antoni MH, Schneiderman N, Carver CS, LaPerriere A, Ironson G, Klimas NG, Fletcher MA. Psychosocial modulation of antibody to Epstein-Barr viral capsid antigen and human herpesvirus type-6 in HIV-1infected and at-risk gay men. Psychosomatic Medicine. 1992.

40. Antoni MH, Lutgendorf SK, Cole SW, Dhabhar FS, Sephton SE, McDonald PG, Stefanek M, Sood AK. The influence of bio-behavioural factors on tumour biology: pathways and mechanisms. Nature Reviews Cancer. 2006 Mar;6(3):240.

41. Wall M, Wheeler S. Benefits of the placebo effect in the therapeutic relationship. Complementary Therapies in Nursing and Midwifery. 1996 Dec 1;2(6):160-3.

42. Richardson P. Placebos: their effectiveness and modes of action. Health Psychology. Boston: Springer. 1989; $34-$ 56.

43. Goodwyn ED. Healing Symbols in Psychotherapy. 2018, Bucureşti, Trei.

44. Fish M. Placebo Therapy. Psychology and Life, 12th Edition. New York: Harper Collins.1979.

\section{Correspondence}

Gabriela Monica Assante,

-PhD, Alexandru Ioan Cuza University, Faculty of Psychology and Educational Sciences, Toma Cozma Street 3, Iași, 700554, panzaru.monica@yahoo.com

Submission: 04 apr 2019

Acceptance: 17 june 2019 